\title{
A fatal and metabolic experimental hemorrhagic shock in immature swine ${ }^{1}$
}

Simone Alves dos Santos Ferreira', Ana Cristina de Moraes", Renato Giuseppe Giovanni Terzi"', Evandro Luis Assis Ferreiralv , William Adalberto Silval, Marcos Mello Moreirav

'Fellow Master degree, Postgraduate Program in Sciences of Surgery, Faculty of Medical School, Universidade Estadual de Campinas (UNICAMP), Brazil. Manuscript preparation.

"Biologist, Center for Experimental Medicine and Surgery, Faculty of Medical Sciences, UNICAMP, Campinas-SP, Brazil. Design the protocol, technical procedures, acquisition and interpretation of data, manuscript writing.

I'PhD, Full and Emeritus Professor, Division of Physiology and Metabology, Department of Surgery, Medical School, UNICAMP, Campinas-SP, Brazil. Design the protocol, technical procedures, acquisition and interpretation of data, manuscript writing.

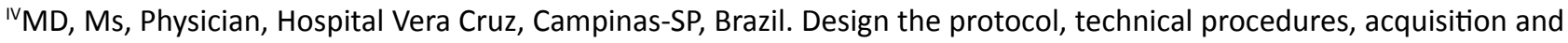
interpretation of data, manuscript writing.

${ }^{\vee}$ PhD, Division of Pneumology, Department of Internal Medicine and, Participant Professor of Postgraduated Program in Sciences of Surgery, Faculty of Medical School, UNICAMP, Campinas-SP, Brazil. Technical procedures, interpretation of data, manuscript wrinting.

\section{Abstract}

Purpose: To use blood lactate $(\mathrm{BL})$ as an end-point metabolic marker for the begin resuscitation of volume replacement in experimental hemorrhagic shock.

Methods: Group I ( $n=7)$ was not bled (Control). Animals in Group II $(n=7)$ were bled to a MAP of $30 \mathrm{mmHg}$ in thirty minutes. Hemodynamic and metabolic data were recorded at Baseline, at 30, 60 and 120 minutes after Baseline. The animals were intubated in spontaneous breathing $\left(\mathrm{F}_{1} \mathrm{O}_{2}=0.21\right)$ with halothane.

Results: Group I all survived. In Group II all died; no mortality occurred before a BL $<10 \mathrm{mM} / \mathrm{L}$. Beyond the end-point all animals exhibited severe acidemia, hyperventilation and clinical signs of shock. Without treatment all animals died within $70.43 \pm 24.51 \mathrm{~min}$ of hypotension shortly after reaching an average level of BL $17.01 \pm 3.20 \mathrm{mM} / \mathrm{L}$.

Conclusions: Swine's breathing room air spontaneously in hemorrhagic shock not treated a blood lactate over $10 \mathrm{mM} / \mathrm{L}$ results fatal. The predictable outcome of this shock model is expected to produce consistent information based on possible different metabolic and hemodynamic patterns as far as the type of fluid and the timing of resuscitation in near fatal hemorrhagic shock.

Key words: Shock, Hemorrhagic. Lactic Acid. Swine. 


\section{Introduction}

Animal experiments done in laboratories suffer some limitations when compared to patients in hemorrhagic shock. The removal of blood through a catheter targeted to a given volume or to a mean arterial pressure (MAP) may not simulate a fulminating bleeding that has ceased immediately ${ }^{1}$ or a persistent bleeding by an open vessel, a situation known as uncontrolled bleeding ${ }^{2}$. In spite of these limitations, the use of a model of hemorrhagic shock is valuable, in order to simulate most of the serious acute hemorrhages observed clinically and to induce a low perfusion state for a period long enough to produce hemodynamic and metabolic effects that may lead ultimately to the death of the animal.

The shock model standardized by Wiggers ${ }^{3}$, and modified later on, has been used extensively. In this model the volume removed is controlled by the level of a previously established MAP and maintained by removal or transfusion of blood through a vascular catheter.

Our main interest was to compare different solutions to resuscitate animals submitted to a standardized shock model. When trying to establish an experimental model, we observed that there was a great variability in the hemodynamic and metabolic pattern in our anesthetized animals when submitted to a pressure controlled hemorrhagic shock. Thus, some animals presented respiratory arrest, or cardiac arrhythmia followed by bradycardia and electromechanical dissociation, finally dying in irreversible cardiac arrest. These findings have been previously reported ${ }^{4}$. On the other hand, surviving animals, presented variable changes of their metabolic profile in blood, expressed by base deficit (BD) or lactate. The same happened when a previously established volume of blood (based on body weight) was removed. In other words, surviving animals in hemorrhagic shock, driven, either by pressure or by predetermined volume, did not exhibit systematic and reproducible significant changes in base deficit or lactate levels in the blood.

Our hypothesis for these findings was that different levels of depth of anesthesia could influence the MAP and tissue perfusion.

We theorized that in hemorrhagic experimental shock under anesthesia two factors are responsible for the reduction of the MAP of the animal: the first factor is hypovolemia produced by bleeding. The second factor is the influence of the anesthetic agents on systemic vascular resistance and myocardial contractility. It is known that when hemodynamically unstable trauma patients in hemorrhagic shock are submitted to anesthesia they are at increased risk because the additional circulatory depression that can induce irreversible shock and death.

Depending on the depth of anesthesia, the animal model with a controlled MAP may produce animals with variable degrees of tissue perfusion and consequently, variable metabolic damage to cells, tissues and organs ${ }^{5-9}$. The non-homogeneity of the physiologic insult in different animals can result in nonhomogeneous outcome to different regimes of volume replacement when studying treatment of hemorrhagic shock. We are suggesting that the level and the kind of anesthesia could interfere in MAP especially because was not our aim to compare different anesthetics or anesthesia depth.

Based on these premises, we intended to identify the parameter that could express the global physiologic insult suffered by the animal in shock. Although base deficit, respiratory arrest and cardiac arrhythmia have been previously reported as endpoints before volume replacement ${ }^{10-12}$ we believe that blood 
lactate levels are more specific to express the degree of anaerobiosis induced by a low perfusion state.

To our knowledge this parameter has never been used before as an endpoint in a model of experimental hemorrhagic shock in order to establish the starting point to resuscitation with fluids.

\section{Methods}

This study was approved by Ethic Commission on Animal Experimentation, Biologic Institute, Universidade Estadual de Campinas (no 163-1).

Large-White piglets $(n=14)$, weighing between 20 and 25 kilograms were used in this study. The animals were pre-medicated with $10 \mathrm{mg} / \mathrm{kg}$ IM ketamine and $0.5 \mathrm{mg} \mathrm{IM}$ atropine. They were anesthetized by induction and were maintained, first by face mask and afterwards by endotracheal tube using nebulized $1 \%$ Halothane and always breathing room air $\left(\mathrm{F}_{1} \mathrm{O}_{2}=0.21\right)$ spontaneously.

A number 5 Swan-Ganz catheter was introduced through the right femoral vein and the tip of the catheter located in the pulmonary artery. A number $6 \mathrm{~F}$ polyethylene catheter was introduced through the femoral artery and the tip located in the abdominal aorta. A number $8 \mathrm{~F}$ urinary catheter was guided to a central position through a left jugular access. The adequate position of all catheters was confirmed by the pattern of the pressure curves.

Each catheter was filled with heparinized solution $(0.9 \%$ saline with 5 units heparin per $\mathrm{ml}$ ). The femoral artery catheter and the Swan-Ganz catheter were connected to transducers Medex. Pressures and cardiac output were measured and registered with a hemodynamic recorder. All pressures were recorded with the animal in supine position with the zero line calibrated to the midthoracic line. Calibration was performed with a standard mercury column.

Cardiac output was measured by thermo-dilution with injection of cold dextrose in water at $5 \%$ below 5 degrees Celsius. Central temperature was recorded through the Cardiac Output Monitor by direct recording of the pulmonary artery temperature.

Blood samples were collected from the femoral artery (arterial) and pulmonary artery (mixed venous). After discarding dead space fluid in arterial and mixed venous blood lines, samples were collected in heparinized syringes for determination of blood gases and blood lactate. Blood samples were immediately processed for blood gases at 38 degrees Celsius and corrected for central body temperature of the animal. Blood lactate was measured with a fast analyzer that permits reading lactate values within one minute in a single drop of blood.

The piglets were divided in two groups: Group I $(n=7)$ Control - Anesthesia and placement of all catheters, but no bleeding. Blood samples were collected and hemodynamic variables were recorded from baseline up to 120 minutes of light anesthesia.

Group II ( $n=7)$ Hypotension - Anesthesia and placement of all catheters. The animals were bled down to a mean arterial pressure of $30 \mathrm{mmHg}$. Blood pressure was maintained at that level by reducing the Halothane concentration to $0.5 \%$ and removing additional blood. Blood lactate level was recorded at short intervals and no further intervention was made. Time of death was recorded after beginning of hypotension and after the last measured lactate. Lactate was collected at short intervals after induction of hypotension, the values recorded but no other interventions was made. Time of death was recorded after 
beginning of hypotension and after the last measured lactate.

\section{- Results} procedure.

In Group I, all animals survived the

In Group II, all animals died. The animals with a MAP kept constant, around $30 \mathrm{mmHg}$ exhibited great variability in the time to reach the levels of an elevated lactate that culminated with the death of the animals.

The animals after reaching a MAP of $30 \mathrm{mmHg}$ exhibited a prompt increase in mean arterial pressure as soon as halothane was reduced from 1 to $0.5 \%$, so that it was possible to remove an additional amount of blood. This maneuver was essential to induce an early low perfusion state and lactic acidosis.

The results of blood lactate in the animals of the Control Group are presented in the Table 1.

Table 1 - Blood lactate in Control Group I - Control.

\begin{tabular}{lllll} 
& $\begin{array}{l}\text { Lactate } \\
\text { Bnimal }\end{array}$ & $\begin{array}{l}\text { Lactate } \\
\mathbf{3 0}\end{array}$ & $\begin{array}{l}\text { Lactate } \\
\mathbf{6 0}\end{array}$ & $\begin{array}{l}\text { Lactate } \\
\mathbf{1 2 0 ^ { \prime }}\end{array}$ \\
\hline Ctrl 1 & 1.3 & 1.0 & 1.0 & 1.0 \\
Ctrl 2 & 1.1 & 1.1 & 0.9 & 1.1 \\
Ctrl 3 & 1.6 & 2.3 & 1.9 & 1.9 \\
Ctrl 4 & 2.4 & 1.8 & 1.7 & 1.9 \\
Ctrl 5 & 2.0 & 2.3 & 2.3 & 2.0 \\
Ctrl 6 & 3.0 & 2.7 & 2.2 & 1.9 \\
Ctrl 7 & 2.0 & 1.8 & 2.0 & 1.4 \\
& & & & \\
mean & 1.91 & 1.86 & 1.71 & 1.60 \\
sd & 0.65 & 0.63 & 0.56 & 0.42 \\
\hline
\end{tabular}

The Control Group maintained levels of constant lactate during the period of 120 minutes, without any statistical difference among the different times: Baseline, 30, 60 and 120 minutes after baseline.

Conversely all animals submitted to the bleeding reached a lactate levels above 10 $\mathrm{mM} / \mathrm{L}$.

The lactate was monitored at variable intervals during the hypotensive period as shown in Table 2. Blood lactate levels determined at baseline before bleeding did not differ statistically from the baseline levels measured in the Control Group. The data presented in Table 2 shows the evolution of blood lactate levels during persistent severe hypotension, as well as the last blood lactate level registered before death, time of hypotension before death and survival time of each animal after reaching a blood lactate over $10 \mathrm{mM} / \mathrm{L}$ (Figure 1). 
Table 2 - Blood lactate in Group II - Hypotension.

\begin{tabular}{|c|c|c|c|c|c|c|c|c|}
\hline Animal & $\begin{array}{l}\text { Lactate } \\
\text { Baseline }\end{array}$ & $\begin{array}{l}\text { Lactate } \\
15^{\prime}\end{array}$ & $\begin{array}{l}\text { Lactate } \\
30^{\prime}\end{array}$ & $\begin{array}{l}\text { Lactate } \\
45^{\prime}\end{array}$ & $\begin{array}{l}\text { Lactate } \\
60^{\prime}\end{array}$ & $\begin{array}{l}\text { Lactate } \\
90^{\prime}\end{array}$ & $\begin{array}{l}\text { Time of } \\
\text { Death (1) }\end{array}$ & $\begin{array}{l}\text { Survival } \\
\text { Time (2) }\end{array}$ \\
\hline Shk 1 & 2 & -- & 5.1 & -- & 8.4 & 21.1 & 95 & 5 \\
\hline Shk 2 & 3.7 & -- & 11.9 & -- & 20.7 & & 90 & 60 \\
\hline Shk 3 & 1.3 & -- & 2.4 & 4.3 & 7.9 & 13.3 & 97 & 7 \\
\hline Shk 4 & 2.5 & -- & 10.7 & 18.5 & & & 46 & 16 \\
\hline Shk 5 & 2.1 & -- & 9.1 & 14.5 & 16.7 & & 65 & 20 \\
\hline Shk 6 & 1.2 & 3.7 & 5.7 & -- & 14.6 & & 65 & 5 \\
\hline Shk 7 & 2.7 & 5.2 & 14.1 & & & & 35 & 5 \\
\hline mean & 2.21 & 4.45 & 8.43 & 12.43 & 13.66 & 17.20 & 70.43 & 16.86 \\
\hline sd & 0.86 & 1.06 & 4.18 & 7.32 & 5.49 & 5.52 & 24.51 & 19.96 \\
\hline
\end{tabular}

(1) Time of death after beginning of hypotension of $30 \mathrm{mmHg}$.

(2) Survival time after reaching a blood lactate higher than $10 \mathrm{mM} / \mathrm{L}$.

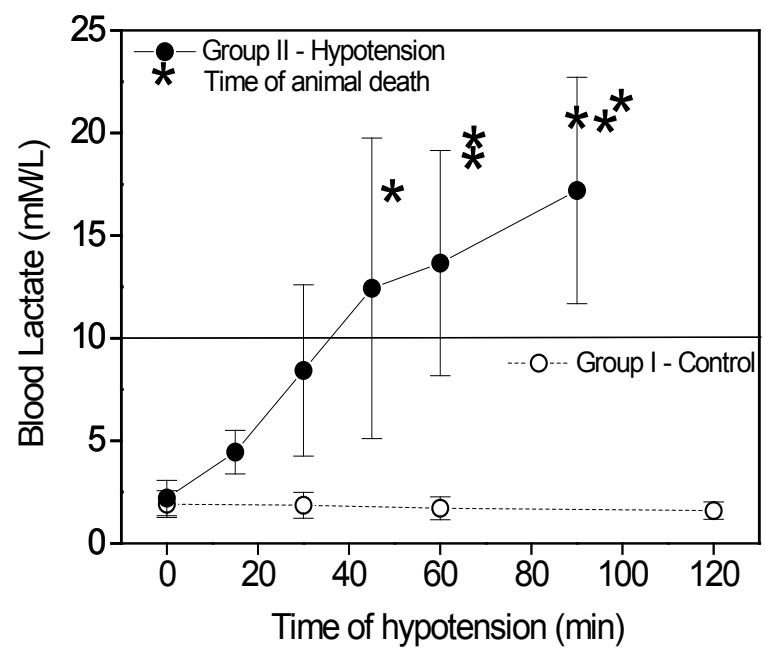

Figure 1 - Blood lactate levels in all hypotensive animals reached values above $10 \mathrm{mM} / \mathrm{L}$.

In these seven animals, we were able to produce an early metabolic response, uniform and systematic. Despite the fact that no further bleeding was induced after a MAP had reached $30 \mathrm{mmHg}$, the animals presented progressive hemodynamic deterioration expressed by a progressive reduction of the MAP. The animals also presented early metabolic and hemodynamic physical signs of hypo perfusion, such as arrhythmia, tachypnea and ischemic, violaceous spots in the abdominal wall. At this time, invariably, blood lactate was over $10 \mathrm{mM} / \mathrm{L}$ and the animals died soon thereafter (Figure 2). Death occurred because of a progressive fall of MAP and/or ventricular arrhythmia and/or electromechanical dissociation. No animal was resuscitated by means of fluid administration, drugs or chest massage because the objective of this work was to evaluate a fatal model of hemorrhagic shock.

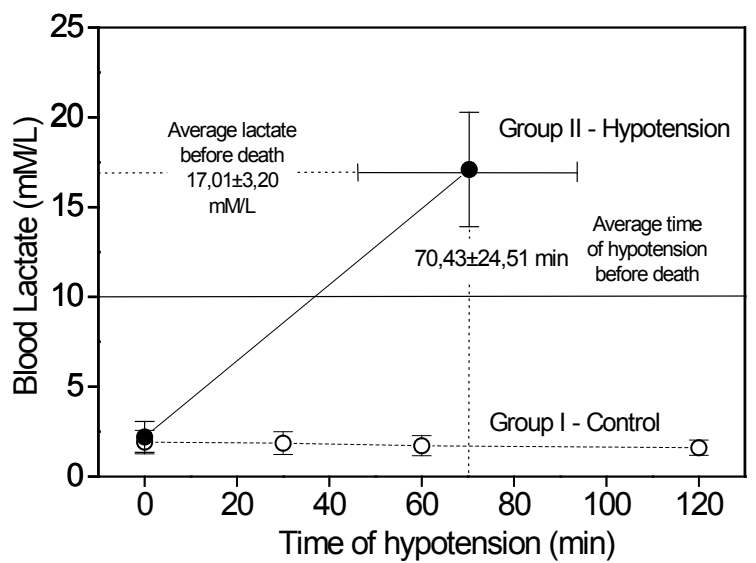

Figure 2 - Average time of hypotension before death was $70.43 \pm 24.51$ minutes in the seven animals submitted bleeding. The last measured blood lactate collected shortly before death was $17.01 \pm 3.20 \mathrm{mM} / \mathrm{L}$. 
Curiously, in this phase of severe hemodynamic instability it was possible to observe a more accentuated reduction of the diastolic than of the systolic pressure.

Outcome of the animals at this time was variable, but correlation between the levels of endpoint lactate and survival time, although suggestive, lacks statistical significance. In fact, all animals died and all presented clinical signs of shock and a blood lactate that varied from 13.3 to $21.12 \mathrm{mM} / \mathrm{L}$ shortly before death (Table 3).

Table 3 - Metabolic variables in Group I (Control) and Group II (Hypotension).

\begin{tabular}{lllll} 
& \multicolumn{2}{l}{$\begin{array}{l}\text { Group I (Control) } \\
n=7\end{array}$} & & \multicolumn{2}{l}{ Group II (Hypotension) } \\
& Baseline & 120' & Baseline & Lac max \\
\hline $\mathrm{pHa}$ (units) & $7.42 \pm 0.05$ & $7.43 \pm 0.04$ & $7.39 \pm 0.06$ & $7.21 \pm 0.23$ \\
$\mathrm{PaCO}_{2}(\mathrm{mmHg})$ & $43.70 \pm 4.5$ & $43.90 \pm 6.3$ & $41.7 \pm 4.9$ & $17.2 \pm 8.6$ \\
$\mathrm{BEa}(\mathrm{mM} / \mathrm{L})$ & $3.50 \pm 3.33$ & $4.41 \pm 2.26$ & $2.25 \pm 1.71$ & $-18.34 \pm 5.8$ \\
Lactate $(\mathrm{mM} / \mathrm{L})$ & $1.9 \pm 0.65$ & $1.6 \pm 0.42$ & $2.21 \pm 0.86$ & $17.01 \pm 3.20$ \\
Shed Blood (\% body weight) & $0 \%$ & & $4.84 \%$ & \\
Mortality before end point & $(0 / 10)$ & & $(0 / 10)$ & \\
Mortality within 24h & $(0 / 10)$ & & $(10 / 10)$ & \\
\hline
\end{tabular}

Baseline: Data collected after instrumentation and stabilization.

120': Data collected two hours after baseline.

Lac max: Data recorded when maximum level of blood lactate was recorded before death.

\section{Discussion}

Preliminary studies from our laboratory in immature pigs keeping a low MAP for a fixed period, showed that the mortality of the animals was variable and it depended, fundamentally on the depth of anesthesia. Thus, some animals presented respiratory arrest or bradicardia early in the hypotensive period, coming to die soon after these severe episodes of cardiopulmonary instability. Frequently, there was no association with high lactate levels. On the other hand, surviving animals after several hours of hypotension under deep anesthesia didn't exhibit significant elevation of their lactate levels and finally survived.

The model of resuscitation with volume replacement after an established period of time of hypotension controlled by pressure or by volume is no guarantee of systematic anaerobiosis and mortality of all animals.
Vivaldi et al. ${ }^{13}$ attempting to standardize hemorrhagic shock in dogs, observed that the volume of initial bleeding, as well as the maximum volume removed from the animals did not correlate with the mortality of the animals. They also reported that the duration of the hypotensive period had only relative importance.

Preliminary studies, also accomplished in our laboratory in immature pigs under deep anesthesia where a volume of blood equal to $4 \%$ of the body weight was removed, resulted in high, early mortality with apnea or arrhythmia when the blood was removed in thirty minutes. In order to reduce early mortality the same volume of blood (4\%BW) was then removed in 90 minutes. Even with prolonged time, mortality was still $40 \%$ before reaching 90 minutes of hypotension. On the other hand, most of the animals that survived the 90 minutes period of hypotension did not exhibit severe lactic acidosis and survived 
over 24 hours without treatment after the experiment.

These observations alerted us to the fact that the determinant of death in these animals was multifactorial. The arrhythmia and respiratory arrest in the early phase in both, pressure or volume controlled hypotension, was attributed to a depression of the respiratory center by the association of hypotension and anesthesia. The anesthetic agents, inducing systemic vasodilatation would determine a more pronounced hypotension without, however, reducing tissue perfusion and cellular oxygenation. The expression of hypotension but with non critical tissue perfusion resulted in less severe lactic acidosis.

Dronen et al. ${ }^{10}$ studying young pigs, emphasized the importance of reaching a goal that could express the degree of physiologic insult just as respiratory arrest or cardiac arrhythmia. However, in our experience, this goal, although associated to the death of the animal when not treated, not always it is associated by significant lactic acidosis. Furthermore, attempts to treat an animal in respiratory arrest or exhibiting severe arrhythmia may not be successful, because these are expressions of near-irreversibility of shock.

The need to monitor some variable that expressed cellular damage was placed initially by Dunham et al. ${ }^{14}$ using statistical methods of probability. Using a modified Kaplan-Meier probability statistic these authors compared lactic acidemia and metabolic base deficit with the conventional hemodynamic variables of blood pressure and cardiac output as predictors of outcome demonstrating their superiority. The LD50 for oxygen debt was shown to be $113.5 \mathrm{ml} / \mathrm{Kg}, 12.9 \mathrm{mM} / \mathrm{L}$ for lactate and -18.8 $\mathrm{mM} / \mathrm{L}$ for BD. Of the single-variable predictors, $B D$ was shown to be the highest explained variability. However, a combined prediction from both, lactate and BD, appeared to be superior to the use of either alone.
Davis ${ }^{11}$ and Davis et al. ${ }^{12}$ suggested that base deficit (BD) could be used as indicator of the severity of shock and adequacy of resuscitation when invasive monitoring was not feasible. They observed that, among other variables, BD would be the best parameter to evaluate volume deficit.

Schultz et $a .^{15}$ in order to compare different regimes of volume replacement in the mouse used BD as a sensitive parameter of inadequate perfusion before treatment. Their endpoint was a BD of $13 \pm 1 \mathrm{mM} / \mathrm{L}$ before beginning resuscitation.

In fact, Davis ${ }^{11}$ and Davis et al..$^{12}$ considered that lactate as well as BD would be parameters equally sensitive to express the physiologic damage determined by hemorrhagic shock and to evaluate the response to fluid replacement. They found a strong linear correlation between these two variables $(R=-0.794)$.

The argument that BD is obtained with more easiness than lactate levels, no longer is justified in our model, because lactate can now be easily monitored almost on real time in the field with a hand-held lactimeter. The lactimeter used in our experiments was Accusport ${ }^{\circ}$ which can read the lactate in a single drop of blood ${ }^{16-17}$.

The use of Accusport for the serial blood lactate determinations permitted monitoring anaerobiosis through lactic acidosis at intervals as short as five minutes. We were able to establish that for young swine the cutoff blood lactate of $10 \mathrm{mM} / \mathrm{L}$ can predict death if no treatment is instituted ${ }^{17}$. This model could be criticized because the sample is small, and it is possible that in a larger population we could prove to have untreated survivors that had reached blood lactate over $10 \mathrm{mM} / \mathrm{L}$ and this limitation may not be disregarded.

This protocol permitted the use of this model to evaluate volume replacement with different fluids such as Lactated Ringer, Colloids and hypertonic solution. The endpoint to start 
resuscitation with volume was established as $10 \mathrm{mM} / \mathrm{L}$ lactate in the arterial blood, no matter the amount of removed volume of blood, the level of mean arterial pressure or the duration of the hypotension. In order to simulate human hemorrhagic shock after trauma and to reduce the time of hypotension needed to attain this endpoint it is necessary to establish a light level of anesthesia to permit additional bleeding and keeping mean arterial pressure around $30 \mathrm{mmHg}$. This model permits to reach lactate levels above $10 \mathrm{mM} / \mathrm{L}$ in a variable period of 30 to 90 minutes. As a matter of fact, blood lactate of seven animals following this protocol showed that a mean endpoint lactate of $17.01 \pm 3.20 \mathrm{mM} / \mathrm{L}$ was reached shortly before death, occurring $70.43 \pm 24.51$ of hypotension before death.

This is probably a better simulation of the time needed for humans to develop severe, hemorrhagic shock before being treated. The predictable outcome of this shock model is expected to produce consistent information based on possible different metabolic and hemodynamic patterns as far as the type of fluid and the timing of resuscitation in near fatal hemorrhagic shock.

\section{Conclusions}

Swine's breathing room air spontaneously in hemorrhagic shock not treated a blood lactate over $10 \mathrm{mM} / \mathrm{L}$ results fatal. The predictable outcome of this shock model is expected to produce consistent information based on possible different metabolic and hemodynamic patterns as far as the type of fluid and the timing of resuscitation in near fatal hemorrhagic shock.

\section{References}

1. Carroll RG, lams SG, Pryor WHJ, Allison EJJ. Single hemorrhage: a clinically relevant canine model of hemorrhagic shock.
Resuscitation. 1988;16(2):119-26. PMID: 2839881.

2. Capone A, Safar P, Stezoski SW, Peitzman A, Tisherman S. Uncontrolled hemorrhagic shock outcome model in rats. Resuscitation. 1995;29(2):143-52. PMID: 7659867.

3. Wiggers CJ. Physiology of shock. New York: The Commonwealth Fund; 1950.

4. Becker H, Hottenrott C, Seufert RM, Gerstenbergk L. Experiences with the hemorrhagic shock in immature pigs. Res Exp Med (Berl.) 1977;170(2):125-31. PMID: 882709.

5. Chahine R, Cheav SL, Nguyen Van Thoai $M$. Effects of corticoadrenal extract on haemorrhagic shock- induced perturbation of systemic haemodynamics and general metabolism in anesthetized dogs. Arzneimittelforschung. 1990;40(6):654-6. PMID: 2396999.

6. Ahlgren I, Aronsen KF, Björkman I. The effect of halothane on the distribution of cardiac output and organ blood flows in the hemorrhagic, hypotensive dog. Acta Anaesthesiol Scand. 1978;22(2):108-16. PMID: 654851.

7. Farnebo LO, Hallman $H$, Hamberger $B$, Jonsson G. Catecholamines and hemorrhagic shock in awake and anesthetized rats. Circ Shock. 1979;6(2):109-18. PMID: 455594.

8. McGown RG. A technique of anaesthesia in haemorrhagic shock. Illustrative case histories and a discussion. Anaesthesia 1975;30(5):616-23. PMID: 1190400.

9. Fontelles MJ, Mantovani M, Hirano ES, Morandin RC. Investigating the relationship between hemodynamic and metabolic parameters in portal triade occlusion following hemorrhagic shock. Acta Cir Bras. 2005;20(1):22-7. PMID: 15810462.

10.Dronen SC, Stern S, Baldursson J, Irvin C, Syverud S. Improved outcome with early blood administration in a near- fatal model of porcine hemorrhagic shock. Am J Emerg Med. 1992;10(6):533-7. PMID: 1388377.

11.Davis JW. The relationship of base deficit to lactate in porcine hemorrhagic shock and resuscitation. J Trauma. 1994;36(2):168-72. PMID: 8114129.

12.Davis JW, Shackford SR, Holbrook TL. Base deficitas a sensitive indicator of compensated shock and tissue oxygen utilization. Surg Gynecol Obstet. 1991;173(6):473-6. PMID: 
1948606.

13.VivaldiE, MacinelliS, GüntherB.Experimental hemorrhagic shock in dogs: standardization. Res Exp Med. 1983;182(2):127-37. PMID: 6867476.

14.Dunham CM, Siegel JH, Weireter L, Fabian M, Goodarzi S, Guadalupi P, Gettings L, Linberg SE, Vary TC. Oxygen debt and metabolic acidemia as quantitative predictors of mortality and the severity of ischemic insult in haemorrhagic shock. Crit Care Med. 1991;19:231-43. PMID: 1989759.

15.Schultz SC, Hamilton IN, Malcolm DS. Use of base deficit to compare resuscitation with lactated Ringer's solution, Haemaccel, whole blood, and diaspirin cross-linked hemoglobin following hemorrhage in rats. J Trauma. 1993;35(4):619-25. PMID: 8411288.
16. Terzi RGG, Alvim CF, Oliveira WC, Moraes VS, Batista ER. Avaliação de um lactímetro de bolso no choque hemorrágico experimental. Rev Bras Ter Intens. 1998;10:42.

17. Ferreira EL, Terzi RG, Silva WA, de Moraes AC. Early Colloid Replacement therapy in a near-fatal model of hemorrhagic shock. Anesth Analg. 2005;101:1785-91. doi: 10.1213/01.ANE.0000184133.48569.55.

\section{Acknowledgements}

To team of the Center for Experimental Medicine and Surgery, Faculty of Medical Sciences, UNICAMP, especially to biologists Willian Adalberto Silva and Ana Cristina Moraes.

\section{Correspondence:}

Simone Alves dos Santos Ferreira Universidade Estadual de Campinas, Cidade Universitária Zeferino Vaz

Rua Tessália Vieira de Camargo, 126

13083-887 Campinas - SP Brasil

simis.alves@gmail.com

Received: Aug 08, 2017

Review: Oct 10, 2017

Accepted: Nov 09, 2017
Conflict of interest: none

Financial source: FAPESP (Grant 93/5012-1)
${ }^{1}$ Research performed at Center for Experimental Medicine and Surgery, Department of Surgery, Faculty of Medical Sciences, Universidade Estadual de Campinas (UNICAMP), Brazil. 